\title{
Evaluation of Skeletal Metastases in Different Clinical Stages of Breast Cancer Patients
}

\author{
M M Arif Hosen ${ }^{1}$, Md Dayem Uddin ${ }^{2}$, Ipshita Zerin ${ }^{3}$, Nasrin Begum ${ }^{4}$ \\ Pupree Mutsuddy, Sunny Anam ${ }^{6}$, Farjana Shimu ${ }^{7}$, Julekha Sharkar ${ }^{8}$
}

\begin{abstract}
Background: Breast cancer is a common form of cancer among women globally. Bone is the most common site to which breast cancer metastasizes. Between $30 \%$ and $85 \%$ of patients with metastatic breast cancer will develop bone metastases during the course of the disease. Objective: To evaluate the pattern and distribution of skeletal metastases in different clinical stages of breast cancer patients by using Tc-99m MDP bone scan. Materials and methods: A retrospective study was conducted on 305 consecutive female breast carcinoma patients referred for bone scan to Institute of Nuclear Medicine and Allied Sciences, Rajshahi from January 2015 to June 2017. All patients were categorized in one of the three groups; early breast cancer (EBC, comprises clinical stage I and II), locally advanced breast cancer (LABC, clinical stage III) and metastatic breast cancer (MBC, clinical stage IV). Bone scan was performed by an intravenous bolus injection of $20 \mathrm{mCi}$ Tc99m-MDP and bone phase images were taken at three hours after injection of the radiotracer. Results: Out of $\mathbf{3 0 5}$ studied patients, 16 patients (5.25\%) were in EBC group, LABC patients were $173(56.72 \%)$ and MBC were 116 (38.03\%). 98 patients (32.13\%) were positive for skeletal metastases. In 16 EBC patients only 01 patient $(6.25 \%)$ had skeletal metastasis in thoracic spine. Skeletal metastases were $20.81 \%$ in LABC and $52.59 \%$ in MBC group. Thoraco-lumbar spine was the commonest site in both groups followed by ribs, pelvic bones, upper and lower extremities, cervical spine, sternum, scapula and skull bone. Conclusion: Bone scan should be done in all LABC and MBC patients and in symptomatic EBC at disease presentation. Parts of bone to focus are thoracic and lumbar spine in all stages, followed by ribs, pelvis and extremities. Tc-99m MDP bone scan play a major role in early detection of skeletal metastasis in breast cancer patients.
\end{abstract}

Key words: Breast cancer, Tc-99m MDP bone scan, skeletal metastasis.

TAJ 2017; 30: No-2: 47-53

\section{Introduction}

Breast cancer is a common form of cancer among women globally. It's the fifth leading cause of death with an average of 522,000 cases per year. ${ }^{1}$ Currently it is the most leading cause of cancer death with 198,000 deaths per annum which represents $15.4 \%$ of all deaths in developed regions after that of the lung cancer. ${ }^{2}$ In developing countries, it is the first leading cause of death among women with 324,000 deaths which

${ }^{1}$ Assistant Professor, Institute of Nuclear Medicine and Allied Sciences, Rajshahi, Bangladesh.

2 Professor and Head, Department of Clinical Oncology, Barind Medical College, Rajshahi, Bangladesh.

${ }^{3}$ MS Fellow in Electrical Power Engineering, Faculty of Engineering, University of Putra, Malaysia.

${ }^{4}$ Associate Professor, Institute of Nuclear Medicine and Allied Sciences, Rajshahi, Bangladesh.

${ }^{5}$ Assistant Professor, National Institute of Nuclear Medicine and Allied Sciences, Dhaka, Bangladesh.

${ }^{6}$ Assistant Professor, Institute of Nuclear Medicine and Allied Sciences, Bogra, Bangladesh.

${ }^{7}$ Resident, Department of Gynae and Obs, Rajshahi Medical College Hospital, Rajshahi, Bangladesh.

${ }^{8}$ Medical Officer, Department of Radiotherapy, Rajshahi Medical College Hospital, Rajshahi, Bangladesh. 
represented $14.3 \%$ of all deaths. ${ }^{2}$ Bone is the most common site to which breast cancer metastasizes. Between $30 \%$ and $85 \%$ of patients with metastatic breast cancer will develop bone metastases during the course of the disease. Bone also represents the first site of metastasis for $26 \%$ to $50 \%$ of patients with metastatic breast cancer. Complications of bone metastasis include bone pain, pathologic fractures (the incidence of which ranges from 16\% to $60 \%$ ), hypocalcaemia, and spinal cord compression, any of which can profoundly impair quality of life. $^{3}$

Bone scan continues to be the most widely used radionuclide technique for investigation of skeletal metastasis primarily due to its wide spread availability. ${ }^{4}$ Radiotracer uptake depends on local blood flow, osteoblastic activity and extraction efficiency. ${ }^{5}$ A major advantage of radionuclide bone scanning is that imaging of the whole skeleton can be performed. This is important as metastatic lesions can occur in regions of the appendicular skeleton that are not routinely included in a radiological skeletal survey. ${ }^{6} \mathrm{~A}$ further advantage relates the high sensitivity of bone scan which enables earlier detection of osseous metastases. Many radiopharmaceuticals (radionuclides) have been used in bone scan including technetium-99m bound to methylene diphosphonate. Published sensitivity and specificity rates of bone scan for diagnosis have varied, with sensitivity ranging from $62 \%$ to $100 \%$ and specificity from $78 \%$ to $100 \%$. However, bone scan is generally considered sensitive for detecting bone metastases on whole-body images. ${ }^{7}$

The aim of this retrospective study was to evaluate the pattern and distribution of skeletal metastases in different clinical stages of breast cancer patients by using Tc-99m MDP bone scan.

\section{Materials and Methods}

A retrospective study was conducted on 305 consecutive female breast carcinoma patients referred for bone scan to Institute of Nuclear Medicine and Allied Sciences, Rajshahi from January 2015 to June 2017. The mean age of the patients was $42.7 \pm 12.9$ years (mean \pm SD) with range from 31 to 68 years. Patient's clinical records and bone scan reports were reviewed retrospectively. All patients were categorized in one of the three groups; early breast cancer (EBC, comprises clinical stage I - freely movable tumor on underlying muscle and no suspicious lymph nodes and stage II - as stage I but ipsilateral mobile axillary node/nodes), locally advanced breast cancer (LABC, comprises clinical stage III primary tumor more extensive than stage I, e.g. skin invaded wide of the primary mass or fixation to muscle. Axillary nodes, if present, are fixed; or supraclavicular nodes involved) and metastatic breast cancer (MBC, comprises clinical stage IV extension beyond the ipsilateral chest wall area, e.g. opposite breast or axilla; or distant metastases). In our institute we used SPECT digital dual head gamma camera (e-cam series, Siemens from Germany) with a low-energy high resolution parallel-hole collimator. Bone scan was performed by an intravenous bolus injection of 20 mCi Tc99m-MDP (technetium 99m methylenediphosphonate). Bone phase images were taken at three hours after injection of the radiotracer and the scan time was about 15 minutes for a whole body scan. Whole body scan in anterior and posterior projections were obtained. All scans were interpreted for metastatic deposits by two independent nuclear medicine physicians.

\section{Results}

Out of 305 studied patients, 16 patients (5.25\%) were in EBC group, LABC patients were 173 (56.72\%) and MBC were 116 (38.03\%) (Figure 1). 


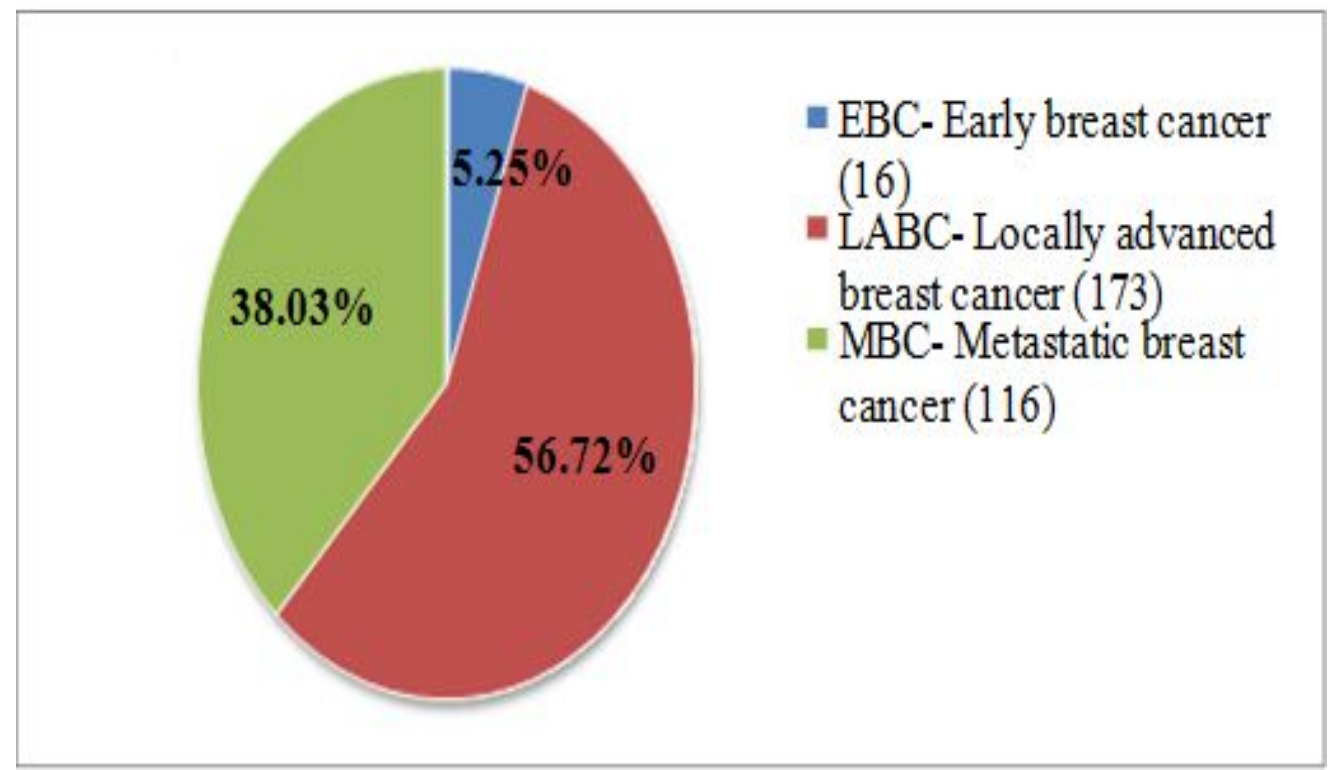

Figure 1: Distribution and percentage of clinical staging at presentation in the study group $(n=305)$

Out of 305 patients 98 patients (32.13\%) were positive for skeletal metastasis, either multiple or solitary lesion. In 16 EBC patients only 01 patient (6.25\%) had skeletal metastasis in thoracic spine. 20.81\% (36/173) had skeletal metastasis in LABC patients and 52.59\% (61/116) had skeletal metastasis in MBC group (Figure 02)

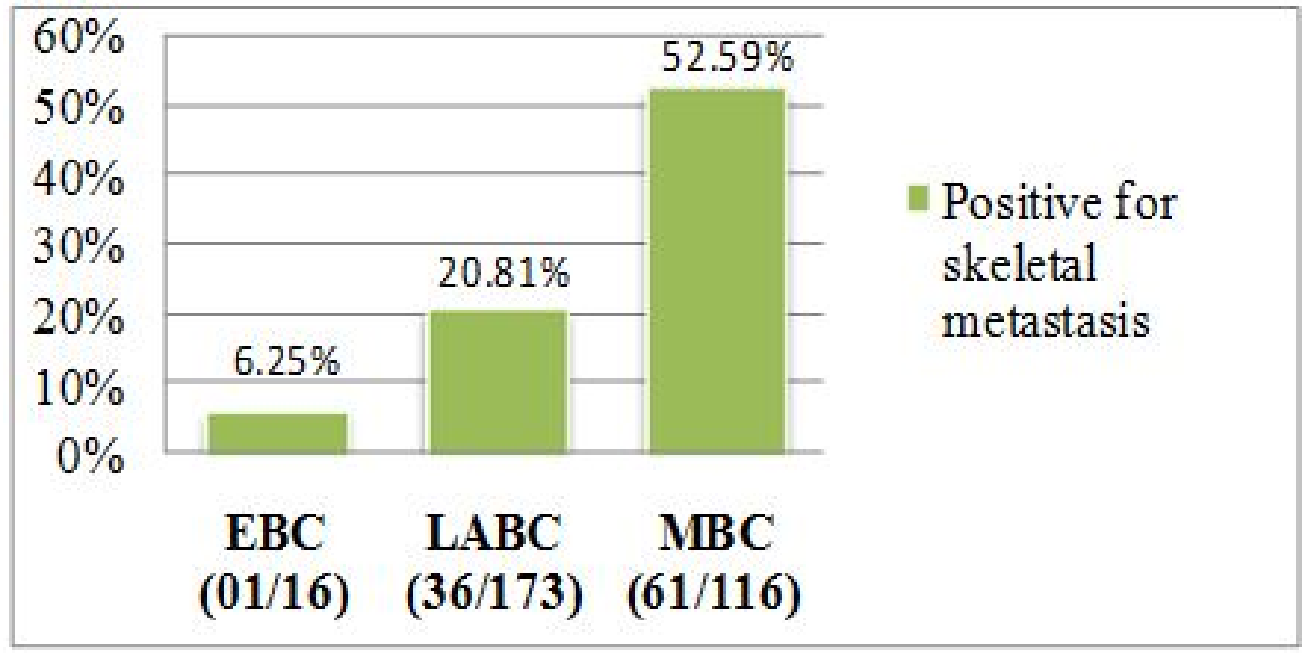

Figure 2: Distribution and percentage of positive skeletal metastases in different clinical stages.

Out of 36 patients in LABC group with skeletal metastasis, highest number was noted in thoraco-lumbar spine (88.89\%). Other sites of metastases were in ribs (72.22\%), pelvic bones (63.89\%), upper extremities (50.00\%), lower extremities (47.22\%), cervical spine (27.78\%), sternum (8.33\%) and skull bone (2.78\%) (Figure 3). 


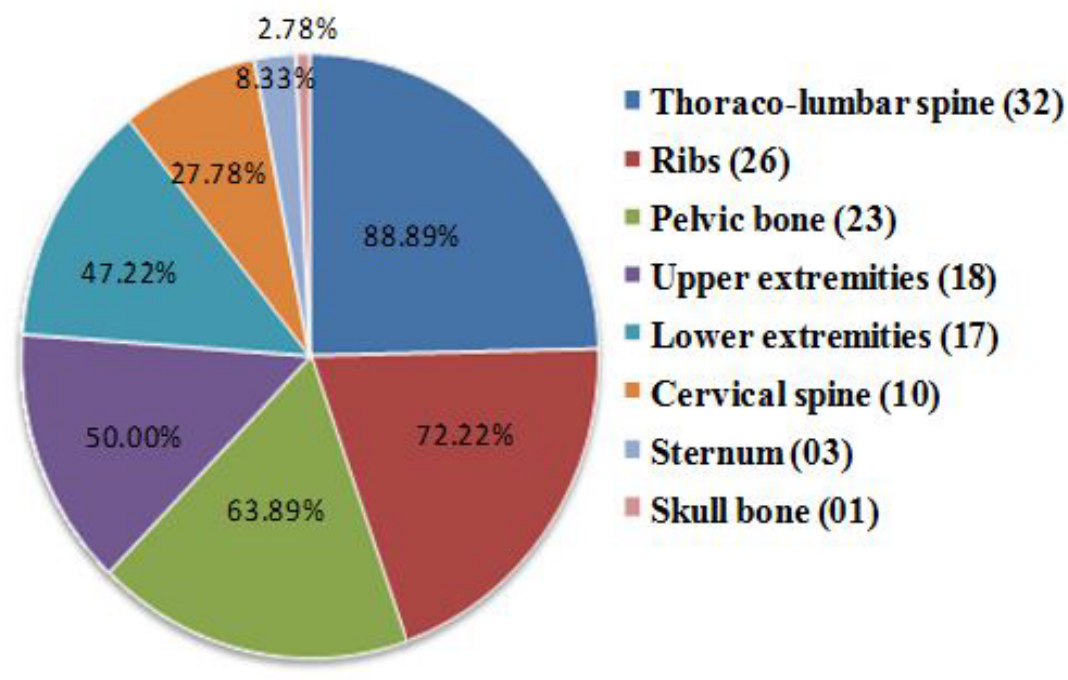

Figure 3: Distribution of skeletal metastases in frequency and percentage by anatomical sites in LABC group ( $\mathrm{n}=36)$

Out of 61 patients in MBC group with skeletal metastasis, maximum number was noted in thoracolumbar spine (73.78\%), followed by pelvic bones (57.38\%), ribs (52.46\%), upper extremities (47.54\%), lower extremities (40.98\%), cervical spine (24.59\%), sternum (13.11\%), scapula (4.91\%) and skull bone (3.28\%) (Figure 4).

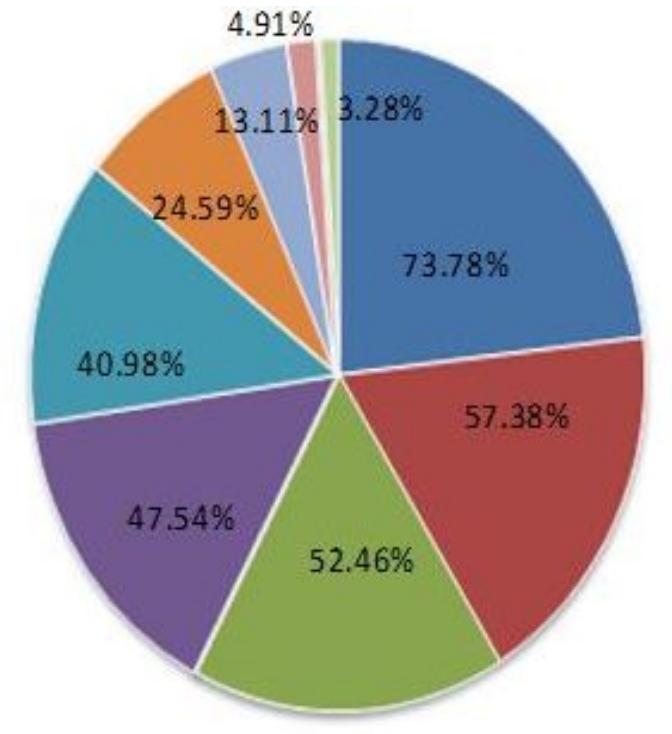

- Thoraco-lumbar spine (45)

- Pelvic bone (35)

- Ribs (32)

- Upper extremities (29)

- Lower extremities (25)

= Cervical spine (15)

- Sternum (08)

- Scapula (03)

"Skull bone (02)

Figure 4: Distribution of skeletal metastases in frequency and percentage by anatomical sites in MBC group $(n=61)$ 


\section{Discussion}

Metastasis of malignant neoplasms to bone is common with metastases being far more prevalent than primary bone malignancies. ${ }^{4}$ Indeed, bone is the third most common organ affected by metastasis, surpassed only by the lungs and liver, and is the most common site of distant metastasis from primary breast cancer. ${ }^{8}$ Breast cancer has a propensity for metastasizing to bone and is, therefore, termed osteotropic. ${ }^{6}$ The selective deposition and proliferation of discrete circulating malignant cells within the skeleton relates to the "seed and soil" hypothesis of tumour biology originally conceptualized by Stephen Paget in the late $19^{\text {th }}$ century. In accordance with this hypothesis, the bone environment represents a "fertile soil" in which some, but not all, cancer cell types (seeds) can flourish. Metastasis to bone can occur via direct extension, arterial or venous spread with the latter representing the most common form. Once in the circulation, entry of the cancer cells into the venous circulation of the bone marrow is facilitated by the slow blood flow and the fact that hematopoietically active bone marrow is well vascularized. ${ }^{8}$ Adhesion molecules produced by tumour cells bind to marrow stromal cells and bone matrix. ${ }^{9}$ The normal remodeling process of bone provides chemotactic and growth factors which support these cancer cells once in place. Skeletal metastases can be osteoblastic (bone forming) or osteolytic (bone destructive); however, a combination of both processes occurs in most cancers ${ }^{8}$. Osteoblastic lesions are usually seen in breast cancer. ${ }^{10}$ The vertebrae, pelvis, ribs and the ends of long bones are preferred destinations of metastases because of their high red marrow content ${ }^{11}$. Within the spine, most metastases are located in the lumbar spine, less frequently in the thoracic spine, and rarely in the cervical spine (52\%, 36\% and $12 \%$ respectively). ${ }^{12}$

Tc-99m MDP bone scan is a highly sensitive technique in detecting metastatic deposits within skeleton, $50 \%$ to $80 \%$ more sensitive than radiographs. The high sensitivity of this technique is based on physiological basis for preferential uptake of methyl diphosphonate, which identifies as little as $5-15 \%$ alteration in local bone turnover.
Delineation of a lytic lesion by conventional radiology requires a minimum size of $1 \mathrm{~cm}$ and a focal loss of at least $50 \%$ of bone mineral, while at least $30 \%$ increase in bone mineral content is essential to appreciate sclerotic lesion. That's why bone scan may pick up bone metastases up to 18 months earlier than conventional radiology, with an average lead of 4 months. The usual appearance of skeletal metastases on bone scan is focal hot spot; however, rarely focal cold defects are also noted. ${ }^{13}$

In our study out of 305 patients, 16 patients (5.25\%) were in EBC group, LABC patients were 173 (56.72\%) and MBC were 116 (38.03). 32.13\% patients (98/305) were positive for skeletal metastasis, either multiple or solitary lesion. M S Afzal et al. (2009) ${ }^{13}$ found positive bone scan in $38 \%$ patients out of 465 breast cancer patients. Skeletal metastases were seen in $23.42 \%$ (241/1029) patients in a study by Doddala $S$ et al. (2016) ${ }^{14}$ Kotb M.H et al. (2013) ${ }^{15}$ found positive bone scan in $18.2 \%$ patients out of 450 . In present study out of 16 EBC patients only 01 patient $(6.25 \%)$ had skeletal metastasis and it was in the thoracic spine. LABC patients had 20.81\% (36/173) skeletal metastasis and 52.59\% (61/116) in MBC group. Results were almost similar with other studies. Doddala S et al. (2016) ${ }^{14}$ found skeletal metastasis in $10.06 \%$ patients in EBC, $25.6 \%$ in LABC and $63.93 \%$ in MBC in a study of 1029 breast cancer patients. In a study done on 1307 patients (1972-1980), skeletal metastases were $15.6 \%$ in $\mathrm{EBC}$ and $24 \%$ in LABC group. A retrospective study by Morris, et al. $(2009)^{16}$ showed that bone metastasis is not common among patients with early stage disease (42 of 266 patients; 15.8\%). Rajiv S et al. (1999) ${ }^{17}$ found skeletal metastases in 3\% patients in EBC and $30 \%$ in LABC group in a study of 250 patients. Mistruku K et al. (2001) ${ }^{18}$ found $34.04 \%$ patients in MBC group in a study of 5538 patients.

Out of 36 patients in LABC group with skeletal metastasis, highest number was noted in thoracolumbar spine (88.89\%), followed by ribs (72.22\%), pelvic bones (63.89\%), upper extremities (50.00\%), lower extremities (47.22\%), cervical spine (27.78\%), sternum (8.33\%) and 
skull bone (2.78\%). Out of 61 patients in MBC group with skeletal metastasis, maximum number was noted in thoraco-lumbar spine (73.78\%), followed by pelvic bones (57.38\%), ribs (52.46\%), upper extremities (47.54\%), lower extremities (40.98\%), cervical spine (24.59\%), sternum $(13.11 \%)$, scapula (4.91\%) and skull bone (3.28\%). The overall pattern and distribution were almost similar to other studies. M S Afzal et al. $(2009)^{13}$ found in patients having multiple skeletal lesions, highest number was in spine $(84.5 \%$ most common in thoracolumbar), followed by ribs (55.5\%), pelvis $(37.3 \%$ - most frequent in iliac bone), skull (32\%), scapula (27.3\%), sternum (26.4\%), femur (19.1\%), humerus (14.5\%), clavicle (3.6\%) and tibia (0.9\%). In another study of patients with breast cancer (Musat $\mathrm{E}$ et al. 1999) ${ }^{19}$, out of 23 subjects with skeletal metastases, 16 cases were having multiple secondaries; the most involved site was the ribs (18 cases), followed by the spine (in 17 cases), iliac bone ( 9 cases), the femur ( 7 cases), the skull (3 cases). In spine, the more frequently involved vertebrae were dorsal (49.23\%), followed by lumbosacral (26.13\%) and lastly cervical vertebrae (12.3\%).

\section{Conclusion}

Bone scan should be done in all LABC and MBC patients and in symptomatic EBC at disease presentation. Metastases should be suspected in the spine in EBC. Parts of bone to focus are thoracic and lumbar spine in all stages, followed by pelvis, ribs and extremities. Tc-99m MDP bone scan is very cost effective in comparison to other imaging modalities like CT, MRI, and PET and play a major role in early detection of skeletal metastasis in breast cancer patients.

\section{References}

1. Shaukat U, Ismail M, Mehmood N. Epidemiology, major risk factors and genetic predisposition for breast cancer in the Pakistani population. Asian Pac J Cancer Prev 2013;14 (10):5625-9.

2. Ly M, Antoine M, Andre F, Callard P, Bernaudin JF, Diallo DA. Breast cancer in Sub-Saharan African women. Bull Cancer 2011;98 (7):797-806.

3. Galasko CS. Skeletal metastases and mammary cancer. Ann R CollSurgEngl1972; 50:3-28.
4. Cuccurullo V, Cascini GL, Tamburrini O, Rotondo A, Mansi L. Bone metastases radio pharmaceuti cals: an overview. Curr Radio pharm 2013; 6: 4147.

5. Gnanasegaran G, Cook G, Adamson K, Fogelman I. Patterns, variants, artifacts, and pitfalls in conventional radionuclide bone imaging and SPECT/CT. Semin Nucl Med 2009; 39: 380-95.

6. Roberts CC, Daffner RH, Weissman BN, Bancroft L, Bennett DL, Blebea JS, et al. ACR appropriateness criteria on metastatic bone disease. J Am CollRadiol2010; 7: 400-409

7. Hortobagyi GN. Novel approaches to the management of bone metastases in patients with breast cancer. SeminOncol2002; 29:134-44.

8. Bussard KM, Gay CV, Mastro AM. The bone microenvironment in metastasis; what is special about bone? Cancer Metastasis Rev 2008; 27: 4155.

9. Choi J, Raghavan M. Diagnostic imaging and image-guided therapy of skeletal metastases. Cancer Control 2012; 19: 102-12.

10. Rajarubendra $\mathrm{N}$, Bolton $\mathrm{D}$, Lawrentschuk N. Diagnosis of bone metastases in urological malignancies--an update. Urology 2010; 76: 78290.

11. Saha S, Burke C, Desai A, Vijayanathan S, Gnanasegaran G. SPECT-CT: applications in musculoskeletal radiology. $\mathrm{Br} \mathrm{J}$ Radiol 2013; 86: 201-05.

12. Talbot JN, Paycha F, Balogova S. Diagnosis of bone metastasis: recent comparative studies of imaging modalities. Q J Nucl Med Mol Imaging 2011; 55: 374-410.

13. Muhammad Shahzad Afzal, M. SaeedAkhtar, AbubakarShahid, Muhammad Babar Imran, Javaid Irfanullah, Muhammad Aleem Khan, Khan Muhammad, Owais Bin Qadeer, Imran Abdullah. Pattern of Distribution of Metastatic Lesions within Skeleton in Patients with Breast Carcinoma of Faisalabad and its Vicinity A.P.M.C 2009; 3(1):1318.

14. Doddala S, Suryadevara A, Chinta S, Madisetty A. Incidence and pattern of bone metastases at presentation in Indian carcinoma breast patients. Indian J Cancer 2016; 53:360-2.

15. Kotb M.H, Wafaie A, Hussein M, Darwish $A$, Almarakby, A. Frequency of Bone Metastases with Metabolic Super Scan in Cancer Breast Patients. Egyptian J. Nucl. Med 2013; 7: 18-29.

16. Schrenk $P$, Rieger $R$, Shamiyeh $A$, Wayand $W$. Morbidity following sentinel lymph node biopsy 
versus axillary lymph node dissection for patients with breast carcinoma. Cancer 2000; 88(3):608-14

17. Rajiv S, Pradip G. Staging investigations in patients with breast cancer. Arch surg 1999; 134:551-53

18. Mistruku K, Masataka Y, Fujio K and Etsuro O. Why do breast cancer patient benefit from staging bone scintigraphy? Jpn J Clin Oncol 2001; 31(6):263-69.
19. Muşat E, Stefanescu C, Rusu V. Whole-body bone scintigraphy in the diagnosis and follow-up of the evaluation of breast cancer. Rev Med Chir Soc Med Nat lasi 1999; 103:163-9.

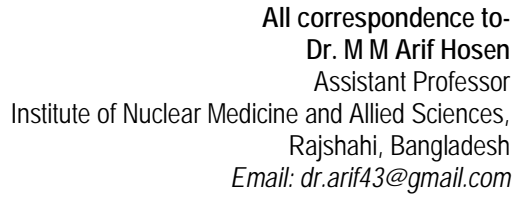

